\title{
Erratum to: The Type I Restriction Enzymes as Barriers to Horizontal Gene Transfer: Determination of the DNA Target Sequences Recognised by Livestock-Associated Methicillin-Resistant Staphylococcus aureus Clonal Complexes 133/ST771 and 398
}

Kai Chen, Augoustinos S. Stephanou, Gareth A. Roberts, John H. White, Laurie P. Cooper, Patrick J. Houston, Jodi A. Lindsay and David T.F. Dryden

\section{Erratum to:}

Chapter 7 in: M.C. Leake (ed.), Biophysics of Infection, Advances in Experimental Medicine and Biology 915, DOI 10.1007/978-3-319-32189-9_7

The original version of this chapter was inadvertently published without the mention of adenine methylation sites for $\mathrm{CC} 1 \mathrm{~S}$. aureus isolates were already determined by the laboratory of Dr. Monk as shown in the MBbio paper in Figure 1. The erratum book and the chapter have been updated with the change.

The updated original online version for this chapter can be found at DOI 10.1007/978-3-319-32189-9_7

K. Chen · A.S. Stephanou · G.A. Roberts · J.H. White · L.P. Cooper · D.T.F. Dryden $(\square)$ EaStCHEM School of Chemistry, University of Edinburgh the King's Buildings,

Edinburgh EH9 3JJ, UK

e-mail: david.dryden@ed.ac.uk

P.J. Houston · J.A. Lindsay $(\bowtie)$

Institute of Infection and Immunity, St George's, University of London,

Cranmer Terrace, London SW17 0RE, UK

e-mail: jlindsay@sgul.ac.uk

Present Address:

K. Chen

Shenyang Research Institute of Chemical Industry, 8 Shenliao Dong Road,

Shenyang Liaoning, People's Republic of China

Present Address:

P.J. Houston

The Pirbright Institute, Ash Road, Pirbright, Woking GU24 0NF, UK 
The authors apologise for this oversight. The correct information is given below:

7.3.4 Identification of Adenines Targeted for Methylation When There Is Ambiguity in the Target Site

Although the orientation of the site and the respective adenine bases that are subject to methylation is clearly determined for CC133/771-1 and CC398-1, this is not always the case using the outlined approach. For example, in our previous paper we assigned the orientation of CC5-1 (SauN315I) as ATC(N) $)_{5}$ CCT (Roberts et al. 2013), but subsequent work using Type I RM systems with S subunits sharing sequence identity to one of the TRDs in CC5-1 clarified that the recognition site of CC5-1 (SauN315I) should be defined in the opposite orientation as AGG(N) $)_{5}$ GAT (unpublished results). Moreover, in our previous work on CC1-1 and CC1-2 we were unable to assign the adenine base in one half of the site that is subject to methylation because there were two possible locations within each target recognition sequence. We have since determined the methylation site using an ATPase assay. Specifically, hemimethylated DNA duplexes containing the target recognition sites for either $\mathrm{CC} 1-1$ or $\mathrm{CC} 1-2$ in which one or other of the possible methylation sites was methylated were designed and generated. A DNA duplex of a similar length lacking the target recognition site was used as a negative control. An ATP-coupled assay was then employed to assess which of the alternative DNA duplexes promote ATPase activity after addition of the reconstituted nuclease for CC1-1 or CC1-2, Fig. 7.4. A duplex containing the unmethylated recognition site will promote the ATPase activity of the complete nuclease. Using this approach we were able to verify that the methylation sites for CC1-1 are CCAY $(\mathrm{N})_{5}$ TTAA and those for $\mathrm{CC} 1-2$ are $\mathrm{CCAY}(\mathrm{N})_{6}{ }_{6} \mathrm{TGT}$ (underlined bases indicate methylation site either on the A shown or on the A on the complementary strand). These methylation sites have also been confirmed recently using single molecule real-time sequencing methods (Monk et al. 2015).

Abstract The Type I DNA restriction-modification (RM) systems of Staphylococcus aureus are known to act as a significant barrier to horizontal gene transfer between $S$. aureus strains belonging to different clonal complexes. The livestock-associated clonal complexes CC133/771 and CC398 contain Type I RM systems not found in human MRSA strains as yet but at some point transfer will occur. When this does take place, horizontal gene transfer of resistance will happen more easily between these strains. The reservoir of antibiotic resistance, virulence and host-adaptation genes present in livestock-associated MRSA will then potentially contribute to the development of newly evolving MRSA clones. The target sites recognised by the Type I RM systems of CC133/771 and CC398 were identified as $\mathrm{CAG}(\mathrm{N})_{5} \mathrm{RTGA}$ and $\underline{\operatorname{ACC}}(\mathrm{N})_{5} \mathrm{RTGA}$, respectively. Assuming that these enzymes recognise the methylation state of adenine, the underlined $\mathrm{A}$ and $\mathrm{T}$ bases indicate the unique positions of methylation. Target methylation points for enzymes from $\mathrm{CC} 1$ were also identified. The methylation points for $\mathrm{CC} 1-1$ are $\mathrm{CCAY}(\mathrm{N})_{5}$ TTAA and those for $\mathrm{CC} 1-2$ are $\mathrm{CCAY}(\mathrm{N})_{6}$ TGT with the underline indicating the adenine methylation site as confirmed recently by Monk et al. (2015) (MBio 6:e00308-15, 2015), which finally clears up the ambiguity noted 
previously (Roberts et al. 2013, Nucleic Acids Res 41:7472-7484) for the half sites containing two adenine bases.

The original article was corrected. 